\title{
ICIS: A Smart Look into the Future
}

\author{
Pranav Rane \\ Student \\ D.J.Sanghvi College of \\ Engineering \\ Mumbai, India
}

\author{
Varun Mhatre \\ Student \\ D.J.Sanghvi College of \\ Engineering \\ Mumbai, India
}

\author{
Khushali Deulkar \\ Asst. Prof. \\ D.J.Sanghvi College of \\ Engineering \\ Mumbai, India
}

\begin{abstract}
ICIS is a prospective product introduced by the Boston-startup LAFORGE that is similar to Google Glass and where it stands against it. Icis is an eye wear which enables user to access their notifications, maps, and various other apps as being developed by Laforge and developers for the same. The features of Icis are enumerated as well as the specifications of the same. Icis allows one to access the smartphone without having to remove it from one's pocket. It accomplishes this using Bluetooth connectivity and creates the interface with the app SocialFlo. Icis boasts high functionality while making a fashion statement, all doing so at a much lower price than its Google made counterpart. Icis has a development program, open to the public to advance the functionality of the product. Finally, Icis is compared to Google Glasses and we take a glance at what we can expect in the future.
\end{abstract}

\section{General Terms}

Augmented Reality

\section{Keywords}

Smart glasses; Laforge; Icis; Product Review; Wearable technology

\section{INTRODUCTION}

The smart phones of today have undoubtedly become an integral part of our lives. They have the processing potential comparable to a vintage supercomputer. We use the countless applications available for Android, iOS, and Windows to make our lives simpler, or simply less boring. Here's where the protagonist of our story makes its entrance. Icis, as defined by Laforge, is a "stylish eyewear that allows one to see updates from a smartphone in one's field of vision". Icis is an optical head-mounted display (OHMD) and is currently under development by the Boston-based startup LAFORGE.

An OHMD is a wearable display that has the capability of reflecting projected images as well as allowing the user to see through it that is augmented reality. Icis is a line of "smartspecs" that look like a conventional pair of eyewear. They relay notifications from your phone and overlay them in your line of sight.

As estimated by the Rochester Optical report the estimates that the wearable technologies market is going to be worth more $\$ 30$ Billion by 2018. In such a scenario a device than alter our smartphone usage has the potential to be huge. Google Glass might have begun the wearable eyewear technological revolution but LaForge and ICIS can take it new level that alter our lifestyle and way we interact with technology.

\section{ANATOMY}

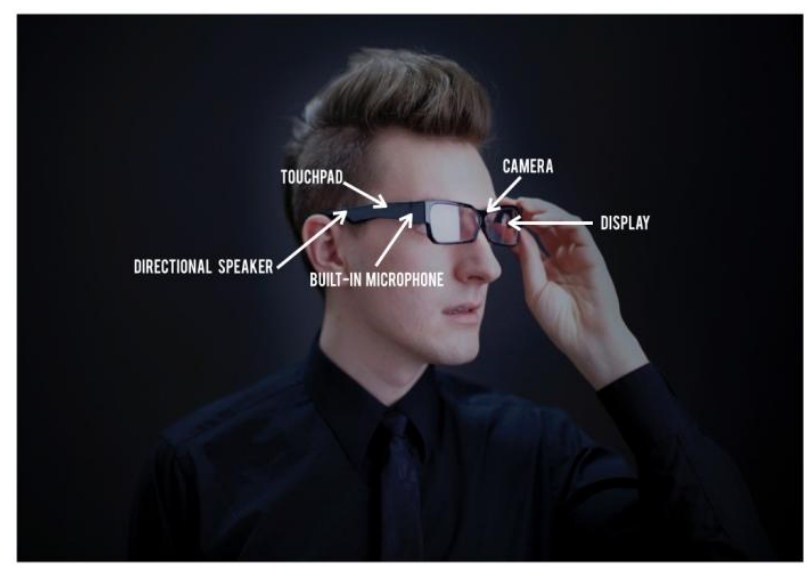

Fig 1: Icis BOLD Anatomy

The Icis has the following components that are listed out and explained in brief. The individual details of each product by Laforge will be discussed under Specifications.

- Camera: A 5MP camera is on the front, on the ridge in between the lens; available in the higher end, Icis BOLD. This will permit video recording and enables capturing pictures.

- Touchpad: The touchpads will most likely be used for navigating through the applications and notifications on the display and access the camera.

- Display: The display is on the lens. The screen is located on the side of the frames, and optical elements in the lens make the image appear in front of the user.

- Built-In Microphone: This is near the lens and is used for audio input by the user, with the help of an on-board chipset. This will enable users to answer calls and have conversations.

- Directional Speaker: The speaker is present near the ear at the ends of the frames on both sides of the frame. This will provide audio feedback which may be used after answering calls.

\section{SPECIFICATION}

There are two variants put forward by Laforge that have been proposed: the Icis and the Icis BOLD. The Icis model has a $1 \mathrm{GHz}$ processor, $512 \mathrm{MB}$ of Ram, a $640 \times 360$ Display, a speaker, and 6 hours of battery life. The Icis BOLD is a beefier version of the regular Icis model with $1 \mathrm{~GB}$ of Ram, an $800 \times 600$ resolution and it has a camera which the Icis model doesn't have. 
Table 1. Icis vs. Icis BOLD.

\begin{tabular}{|c|c|}
\hline ICIS & ICIS Bold \\
\hline $\begin{array}{l}1 \mathrm{GHz} \text { Texas Instruments } \\
\text { Sitara Processor }\end{array}$ & $\begin{array}{lr}1 \mathrm{GHz} \text { Texas } & \text { Instruments } \\
\text { Sitara Processor } & \end{array}$ \\
\hline $1 \mathrm{~GB}$ of memory & 1GB of memory \\
\hline $\begin{array}{l}\text { Kopin Cyberdisplay VGA } \\
\text { LVS } \\
640 x 480 \text { resolution }\end{array}$ & $\begin{array}{l}\text { Kopin Cyberdisplay VGA } \\
\text { LVS } \\
800 \times 600 \text { resolution }\end{array}$ \\
\hline $\begin{array}{l}\text { Bluegiga BT Radio } \\
\text { Bluetooth } 4.0\end{array}$ & $\begin{array}{l}\text { Bluegiga BT Radio } \\
\text { Bluetooth } 4.0\end{array}$ \\
\hline $\begin{array}{l}\text { IambicFLO directional } \\
\text { Speaker } \\
\text { Built-in microphone } \\
\text { On-board audio chipset }\end{array}$ & $\begin{array}{l}\text { IambicFLO directional } \\
\text { Speaker } \\
\text { Built-in microphone } \\
\text { On-board audio chipset }\end{array}$ \\
\hline NFC wireless charging & NFC wireless charging \\
\hline $\begin{array}{l}\text { Compatible with any } \\
\text { Bluetooth-enabled device } \\
\text { running iOS7, Windows } \\
\text { Phone 8, or Android Ice } \\
\text { Cream Sandwich (or later } \\
\text { operating systems) }\end{array}$ & $\begin{array}{l}\text { Compatible with any } \\
\text { Bluetooth-enabled device } \\
\text { running iOS7, Windows } \\
\text { Phone 8, or Android Ice } \\
\text { Cream Sandwich (or later } \\
\text { operating systems) }\end{array}$ \\
\hline N/A & $\begin{array}{l}\text { 5MP Photos } \\
\text { 720p HD video recording }\end{array}$ \\
\hline Sensors include & Sensors include \\
\hline Three-axis gyro & Three-axis gyro \\
\hline Accelerometer & Accelerometer \\
\hline Touchpad & Touchpad \\
\hline
\end{tabular}

\section{DESCRIPTION}

Even though Icis has a relatively conventional appearance, but in actuality, it is a cloud-connected, Bluetooth-enabled device featuring a heads-up display (HUD). It provides smartphone notifications around their field of vision of the user. It is packed into the stylishly contemporary frame and comprises a camera, microphone and speaker that complement the hardware in a user's smartphone.

Users will be able to interact with Icis using touch pads that are temple-mounted. The glasses convert existing smartphone apps into widgets by pairing up with the app and displaying them. The widgets are created with an API, and are used to relay app notifications

\subsection{How it Works}

- The screen will be located in the temples (side of the frames) of the eyewear. A series of optical elements in the lens will make it appear as if the image is focused in front of the user.

- The technology does not use projected images. Instead, something that is known as a virtual image is used. By using a proprietary combination of lenses and coatings that allow the user to see the image in front of them, in focus, no matter where they're looking.

- The electronics of the device are integrated into the temples of the glasses. This device cannot be retrofit into existing frames.
-Icis is an app-driven Bluetooth accessory, which means that it communicates directly with the user's phone, and not to their network.

\subsection{Features}

- The product is designed with prescription lens. At launch, to the device will accommodate people who are near-sighted or far-sighted, or have astigmatism.

- At launch, three designs will be available: A semi-frameless, flat-top, and a wayfarer style. More styles will be offered at a later stage.

-When the device launches, it will work on Windows Mobile, iOS, as well as Android. Icis features a camera, microphone, and speaker to complement the hardware in your phone.

- There is a developer's model available, where they will be developing an app for smartphones: SocialFlo. This turns any app into a widget that Icis can recognize. Any part of a person's field of vision can be used for the widget as long as there are no background images used.

- There are three modes, Normal, Active, and Driving Modes. They transition from one mode to another with the help of an accelerometer.

\subsection{The 3 Modes}

\subsubsection{Normal Mode}

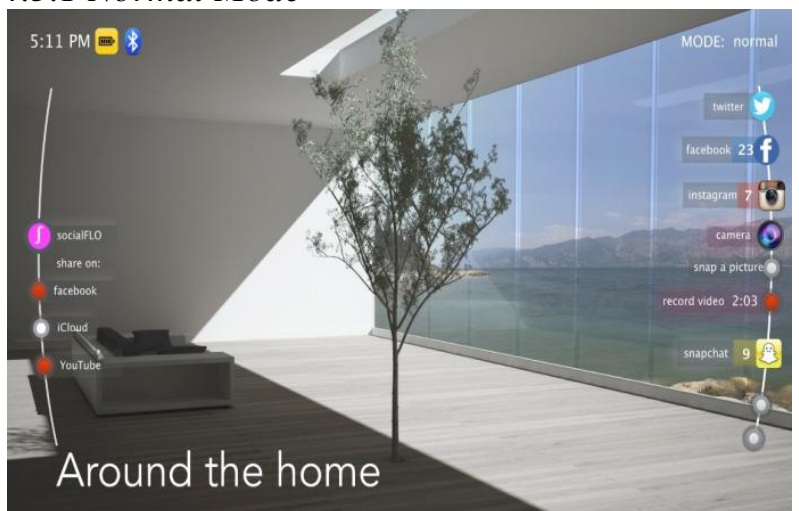

Fig 2: Normal Mode

Normal mode will keep the user up to date on your notifications, and keep the camera just a tap away. This is how Icis will look most of the time. This mode will allow the user to:

- View their notifications

- Take pictures from their camera

- Answer incoming calls

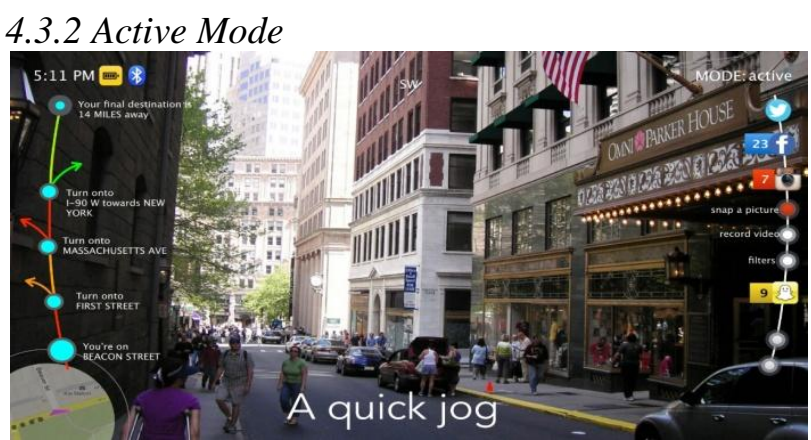

Fig 3: Active Mode 
Once the user begin to move faster than, $3 \mathrm{mph}$ the device may automatically switch into Active mode. Using the app socialFLO, the user can preset their favorite fitness or on-thego widgets to turn on while they're exercising. Here they can:

- Get bicycle routes

- Monitor their fitness performance

- Compete with their friends in real time

\subsubsection{Driving Mode}

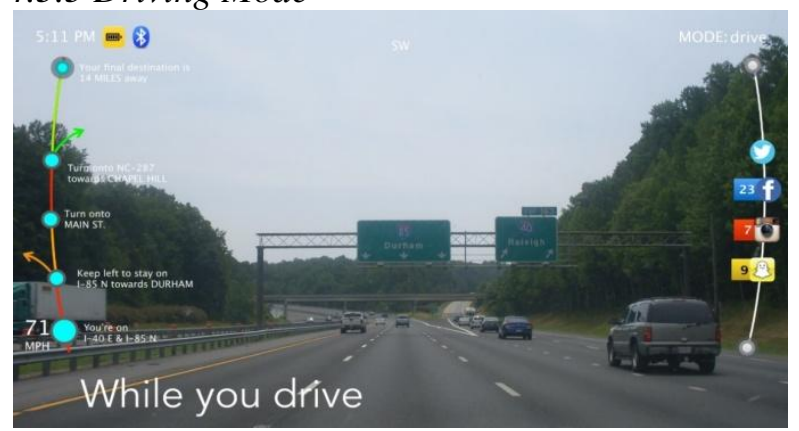

Fig 4: Driving Mode

Icis will automatically switch into Drive mode once the user reach a speed of $20 \mathrm{mph}$. Drive mode will block all notifications and only display the content that's relevant to driving. In this mode, the user will be able to do the following activities:

- Get turn-by-turn directions

- View their speed limits

- Get accident notifications

\section{GOOGLE GLASSES VS. ICIS 5.1 Function}

Google Glass is compatible with any Bluetooth-capable phone with the MyGlass app. Icis is also compatible with any Bluetooth-capable phone via its SocialFlo app. In Google Glass, audio comes from an oval speaker on the inside of the battery pod behind the user's ear. Icis has a built-in microphone, touchpad, audio and wireless charging. Google Glass features voice-activated commands, navigation, wink to take a photo, gesture-controlled actions as well as social media sharing options. To navigate through the Glass menu, users use the touchpad on the side of the device. Once the smart lenses surpass the beta versions, Icis is also expected to feature a camera and high resolution display. The differences in the features of the wearable devices are slim.

Both Google Glass and Icis smart glasses allow wearers to receive mobile phone notifications right in front of their eyes and are designed with the hands-free form factor in mind. Google Glass has the upper hand, in terms of function, with more apps designed specifically for the device. It also has more frequent software upgrades catering to longtime Explorer's preferences. On the other hand, it is important to note that the game changer with Laforge Optical's Icis prescription smart glasses is its design.

\subsection{Design}

Google Glass has turned its geeky aesthetic reputation around by means of a stylish revamp for those lacking a perfect 20/20 vision. The Glass prescription lenses are much more mainstream. They have adopted a Warby Parker-esque design and boast various styles. However, Laforge Optical's Icis smart glasses take this stylish twist a step further by eliminating the miniature computer screen which is seen over the right eye of Glass lenses. In its place, the Icis prescription lenses present information to users using a cloud displayed around the outside field of vision.

\subsection{Price and Release Date}

The Google Glass is reported to launch later in 2014 at a price of around $\$ 600$. Glass does have an edge over Icis since it has been in beta for a reasonable amount of time now. This allows for engineers and designers to tweak the device to perfection ahead of its widespread release. Contrariwise, Laforge Optical is currently offering Indiegogo contributors a pair of its smart specs for only $\$ 420$ at an estimated delivery date of January 2015.

\section{CONCLUSION}

Displays that are mounted on our head, they are the key to innovative hands-free technology. Using this, we can efficiently implement augmented reality. Laforge though not first of its kind in attempting to make a union of the two technologies, it nonetheless shows us the great potential that lies before us. Hardly a decade back, the idea of such a wearable technology, would be considered fantastical, futuristic, or even alien. But, now such devices are not only being designed, but developed into reality. Laforge's Icis shows us that we can integrate technology more closely into our day-to-day lives. It is a step from accessing information, including that of social, from a desktop computer to portable devices in our pockets. By going a step further, we can access these portable devices by integrating them into one of our five senses, effectively leaving them in our pockets.

\section{ACKNOWLEDGMENTS}

We would like to thank our honorable principal Dr. Hari Vasudevan of D. J. Sanghvi College of Engineering and Dr. Narendra Shekokar, Head of Department of Computer Engineering, for giving us the facilities and providing us with a propitious environment for working in college. We would also like to thank S.V.K.M. for encouraging us in such cocurricular activities.

\section{REFERENCES}

[1] http://www.laforgeoptical.com/

[2] http://www.cnet.com/news/icis-its-like-google-glass-butclassy/

[3] http://www.idigitaltimes.com/google-glass-vs-icis-canlaforge-opticals-prescription-smart-lenses-deliver-morefunctionality

[4] http://en.wikipedia.org/wiki/Optical_headmounted_display

[5] http://cdn2.idigitaltimes.com/articles/21960/20140219/go ogle-glass-vs-icis-laforge-opticals-prescription.htm

[6] http://en.wikipedia.org/wiki?curid=42032995

[7] IHS Electronics and Media, 2013. Wearable technology

[8] Cognizant Technologies, 2013. Google Glass: Insurance's next killer app 